\title{
BMJ Open What enables older people to continue with their falls prevention exercises? A qualitative systematic review
}

\author{
Susanne Finnegan, ${ }^{1}$ Julie Bruce, ${ }^{1}$ Kate Seers ${ }^{2}$
}

To cite: Finnegan S, Bruce J, Seers K. What enables older people to continue with their falls prevention exercises? A qualitative systematic review. BMJ Open 2019;9:e026074. doi:10.1136/ bmjopen-2018-026074

- Prepublication history and additional material for this paper are available online. To view these files, please visit the journal online (http://dx.doi org/10.1136/bmjopen-2018026074).

Received 16 August 2018 Revised 18 February 2019 Accepted 21 February 2019

Check for updates

(c) Author(s) (or their employer(s)) 2019. Re-use permitted under CC BY-NC. No commercial re-use. See rights and permissions. Published by BMJ.

${ }^{1}$ Warwick Clinical Trials Unit, University of Warwick, Coventry, UK

${ }^{2}$ RCN RI, Royal College of Nursing Research Institute, Warwick Medical School, University of Warwick, Coventry, UK

Correspondence to Susanne Finnegan; susanne.finnegan@warwick. ac.uk

\begin{abstract}
Objectives To review the qualitative literature that explores the barriers and facilitators to continued participation in falls prevention exercise after completion of a structured exercise programme.

Design A systematic literature review with thematic synthesis of qualitative studies exploring older adults' experiences of continued participation in falls prevention exercise.
\end{abstract}

Data sources Comprehensive searches were conducted in MEDLINE, PSYCHinfo, AMED, ASSIA, CINAHL and EMBASE from inception until November 2017. Additional studies were identified via searches of reference lists and citation tracking of relevant studies.

Eligibility criteria Qualitative or mixed methods studies exploring experiences of community-dwelling older adults (65 years and over) participation in a falls prevention exercise programme including their experience of ongoing participation in exercise after the completion of a structured exercise programme.

Data extraction and synthesis Key characteristics including aim, participant characteristics, method of data collection, underpinning qualitative methodology and analytical approach were extracted and independently checked. Thematic synthesis was used to integrate findings.

Results From 14 studies involving 425 participants, we identified three descriptive themes: identity, motivators/ deterrents and nature of the intervention and one overarching analytical theme: agency.

Conclusions Older people have their own individual and meaningful rationale for either continuing or stopping exercise after completion of a structured falls prevention exercise programme. Exploring these barriers and facilitators to continued exercise is key during the intervention phase. It is important that health care professionals get to know the older person's rationale and offer the best evidence-based practice and support to individuals, to ensure a smooth transition from their structured intervention towards longer-term exerciserelated behaviour.

PROSPERO registration number CRD42017082637.

\section{INTRODUCTION}

Falls remain a global health problem for older people. The WHO recognises falling as the second leading cause of accidental or unintentional injury-related death worldwide. ${ }^{1}$ Thus,

\section{Strengths and limitations of this study}

A systematic review of qualitative data examining the barriers and facilitators to ongoing falls prevention exercise after completion of an exercise programme.

- The methods used ensured that the identified themes were grounded in the data.

- The Critical Appraisal Skills Program (CASP) qualitative checklist was used to assess the trustworthiness, transparency and relevance of the included studies and GRADE-Confidence in the Evidence from Reviews of Qualitative Research (CERQual) was used to assess the credibility of our findings.

- The CASP tool does not claim to be a definitive guide for critical appraisal and there was variability in CASP scores.

intervening to prevent falls is an international priority.

Falls prevention exercise programmes including moderate intensity strength and balance training are effective at reducing rate and risk of falling in older adults, ${ }^{2}$ but exercise needs to be maintained to have a lasting effect. ${ }^{3}$ Falls prevention exercise programmes in both research and health settings tend to be of short duration (<6months), ${ }^{4}$ and uptake and retention to these programmes is challenging. ${ }^{5}$

Numerous quantitative and qualitative studies have been undertaken to understand the reasons behind the problems with uptake and adherence to these structured programmes. ${ }^{6-9}$ There is, however, lack of information about exercise behaviours in the longer term, after completion of falls prevention programmes. To promote lifelong behaviour change and long-term commitment to exercise among older people, it is important to understand the barriers and facilitators to adherence, not only during delivery of structured exercise interventions, but also once the intervention has been delivered.

The aim of this systematic review of qualitative evidence was to explore the barriers and facilitators to continued participation in 
falls prevention exercise once structured falls prevention programmes come to an end.

\section{METHOD}

A qualitative systematic review involves systematically searching for primary qualitative studies which can be integrated using methods such as thematic synthesis. ${ }^{1011}$ The Enhancing transparency in reporting the synthesis of qualitative research (ENTREQ) checklist was used to guide the reporting of this review. ${ }^{12}$

\section{Search strategy and selection criteria}

Comprehensive searches were conducted in MEDLINE, PSYCHinfo, AMED, ASSIA, CINAHL and EMBASE from inception until November 2017. The search strategies are included in online supplementary file 1 . We performed a manual search of reference lists and citation tracking of relevant studies to identify additional papers. The search was restricted to studies published in the English language. Qualitative studies and mixed methods studies that clearly report the qualitative findings separately were included if participants were community dwelling and at least 65 years old or the mean reported age of participants was 65 years or over. Inclusion criteria included studies examining both individual (home-based) or group-based falls prevention exercise programmes with an aim to explore older adults' experiences of ongoing participation in falls prevention exercise after the completion of a structured exercise programme. This included: (1) continuing in a new falls prevention exercise group; (2) continuation of falls prevention exercises independently at home, based on the exercise intervention; or (3) continuing with other/new types of exercise to replace the falls prevention exercises. Studies were excluded if they included participants living in residential or nursing homes, did not include qualitative data collection methods for example, interviews or focus groups and did not generate data about ongoing participation in exercise post-intervention.

\section{Quality appraisal}

The Critical Appraisal Skills Program (CASP) qualitative checklist was used to assess the trustworthiness, transparency and relevance of the included studies. ${ }^{13}$ The tool does not claim to be a definitive guide for critical appraisal but focuses on the key principles underpinning qualitative research. ${ }^{12}$ Studies were not excluded based on the CASP scores, but the information from the assessments were used in the discussion of the overall review.

To complement the CASP checklist we also applied the GRADE-Confidence in the Evidence from Reviews of Qualitative Research (CERQual). ${ }^{14}{ }^{15}$ To ensure a review of this type reasonably represents the area of interest, CERQual provides a systematic and transparent framework for assessing confidence in individual review findings. This framework consists of four components: (1) methodological limitations, (2) coherence, (3) adequacy of data and (4) relevance.
Pairs of review authors (SF and JB/KS) independently assessed each study for quality and credibility and resolved any disagreements through discussion.

\section{Data extraction}

Key characteristics of included studies were extracted by SF and independently checked by JB and KS. Any discrepancies were resolved via discussion. For each study, the following were extracted: author, year of publication, country of origin, stated aim, participant characteristics, method of data collection, underpinning qualitative methodology and analytical approach. Relevant text and illustrative participant quotations from the findings and discussion sections of each study were extracted and discussed before being entered verbatim into NVIVO V.11 to assist in organising the text for analysis.

\section{Data synthesis and analysis}

We used thematic synthesis to integrate findings. ${ }^{11}$ This involved three overlapping, non-linear phases: (1) line by line coding of the findings, (2) organisation of codes into descriptive themes and (3) construction of analytical themes. $^{11}$

One reviewer $(\mathrm{SF})$ performed line by line coding of the primary studies, recording and translating the concepts of participants' experiences of ongoing exercise from one study into another, constantly comparing codes between studies and organising these codes into descriptive themes. KS double coded $20 \%$ of studies, there was broad agreement on the codes and themes and no new codes or themes emerged. As per Thomas and Harden's recommendations, ${ }^{11}$ the descriptive themes and sub-themes were based on the original findings of the included studies. All studies were re-read and reviewed multiple times to ensure that pertinent data were integrated into the descriptive themes appropriately. To 'go beyond' the content of the original studies and construct analytical themes, the review authors discussed the descriptive themes and used mind mapping to create a schematic to expand and explain the themes from the primary studies by identifying cross-cutting conceptual links.

Mind mapping involved using diagrams to visually structure ideas and discussion points about the potential concepts coming from the data. This process enabled the themes to be organised and structured in a more coherent way.

\section{Patient and public involvement}

There was no patient or public involvement in the development of this manuscript.

\section{RESULTS}

\section{Literature search results}

In total, 69 full-text articles were retrieved for review, of which 14 studies were included, involving 425 participants (figure 1).

Study characteristics are described in table 1. 


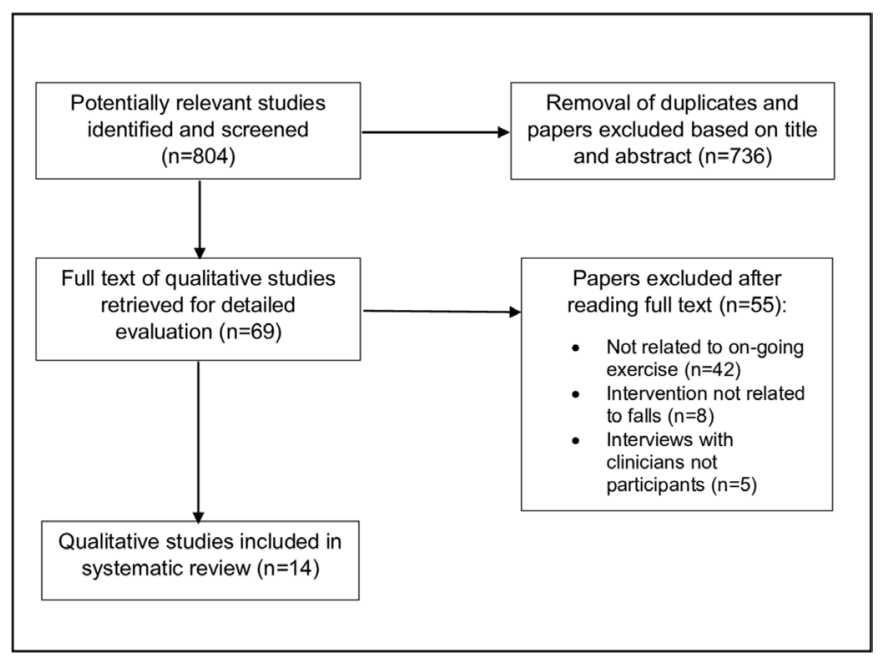

Figure 1 Flow diagram of search and study selection process.

All the exercise interventions in the included studies were group-based exercise programmes. They were delivered as either stand-alone exercise programmes or within a broader multi-factorial falls prevention programme. The aim of two studies was primarily examining issues with the uptake of falls prevention programmes but also explored older people's ideas and suggestions for future programmes and what might be involved in a successful programme including ongoing participation. ${ }^{16} 17$ The studies were undertaken in six countries: seven in the UK, ${ }^{18-24}$ four in Australia/New Zealand, ${ }^{25-28}$ and one each in Norway, ${ }^{29}$ Denmark ${ }^{16}$ and Hong Kong. ${ }^{17}$ One study restricted participant inclusion to men only, ${ }^{27}$ and all studies included community-dwelling adults aged 65 and over (range 65-94 years).

\section{Quality of reporting}

There was some variability in the CASP scores (table 2).

All 14 studies clearly described the aim, selected qualitative research as the appropriate methodology and design to undertake the research and gave a clear statement of their findings. However, only 2/14 (14\%) studies adequately described or considered the relationship between the researcher and the participants; therefore, potential individual researcher biases were not reported. All studies sought ethical approval from a research ethics committee for their research.

Table 3 is a summary of qualitative findings based on the four components of the GRADE-CERQual framework.

For each descriptive theme, studies were assessed for methodological limitations, coherence, adequacy and relevance. We are moderately confident in the themes of identity and motivators/deterrents; thus, it is likely that these review findings are a reasonable representation of the phenomenon of interest. We have high confidence in the nature of the intervention theme, meaning it is highly likely that this finding is a reasonable representation of the phenomenon of interest. ${ }^{14}$

\section{Synthesis}

In identifying the barriers and facilitators to ongoing participation in falls prevention exercise, one overarching analytical theme encapsulating three descriptive themes with sub-themes were derived from the findings of the original 14 studies; they are presented here with narrative exemplars. A schema of the themes was developed using mind mapping and is presented in figure 2.

\section{Descriptive themes}

\section{Theme 1: identity}

This theme and sub-themes describe how the participant's perception of self or their own identity affected their continued participation in exercise.

\section{'Fallers'}

Even though these older adults had all participated in a falls prevention exercise programme, often they did not identify themselves as 'fallers' despite having had a fall. Preventing falls was rarely described as a reason for continued exercise:

A decrease in the likelihood of a fall was infrequently mentioned...also related to this was the perception that the participant was not at risk of falls, possibly in part due to their level of health and fitness, and also in part due to their participation in alternate forms of physical activity $^{25}$

Participants were asked about their attitudes to health service falls prevention interventions...views varied from 'being prepared to try' or 'go along with it', to obvious reluctance...they felt they were not yet in need ${ }^{21}$

Instead, rather than preventing falls, participants described improvements in strength and balance and increased confidence in relation to overall independence, functional improvements and quality of life as important reasons for continued exercise:

A number of benefits were expressed among the participants, including balance improvement...higher confidence in activities of daily living, and greater happiness $^{17}$

The results showed that motivating factors to adhere to recommended exercise were perceived prospects of staying independent, maintaining current health status, and improving physical balance and the ability to walk ${ }^{29}$

\section{'I'm not old'}

Increased age is a risk factor for falling and although the participants identified a link between risk of falling and older age, some did not identify themselves as being old. However, some also did not think of age as a barrier to ongoing exercise:

'...they did not readily identify with the population that they perceived to be the target of the intervention, that is, 'people who fall' or 'old people',25 


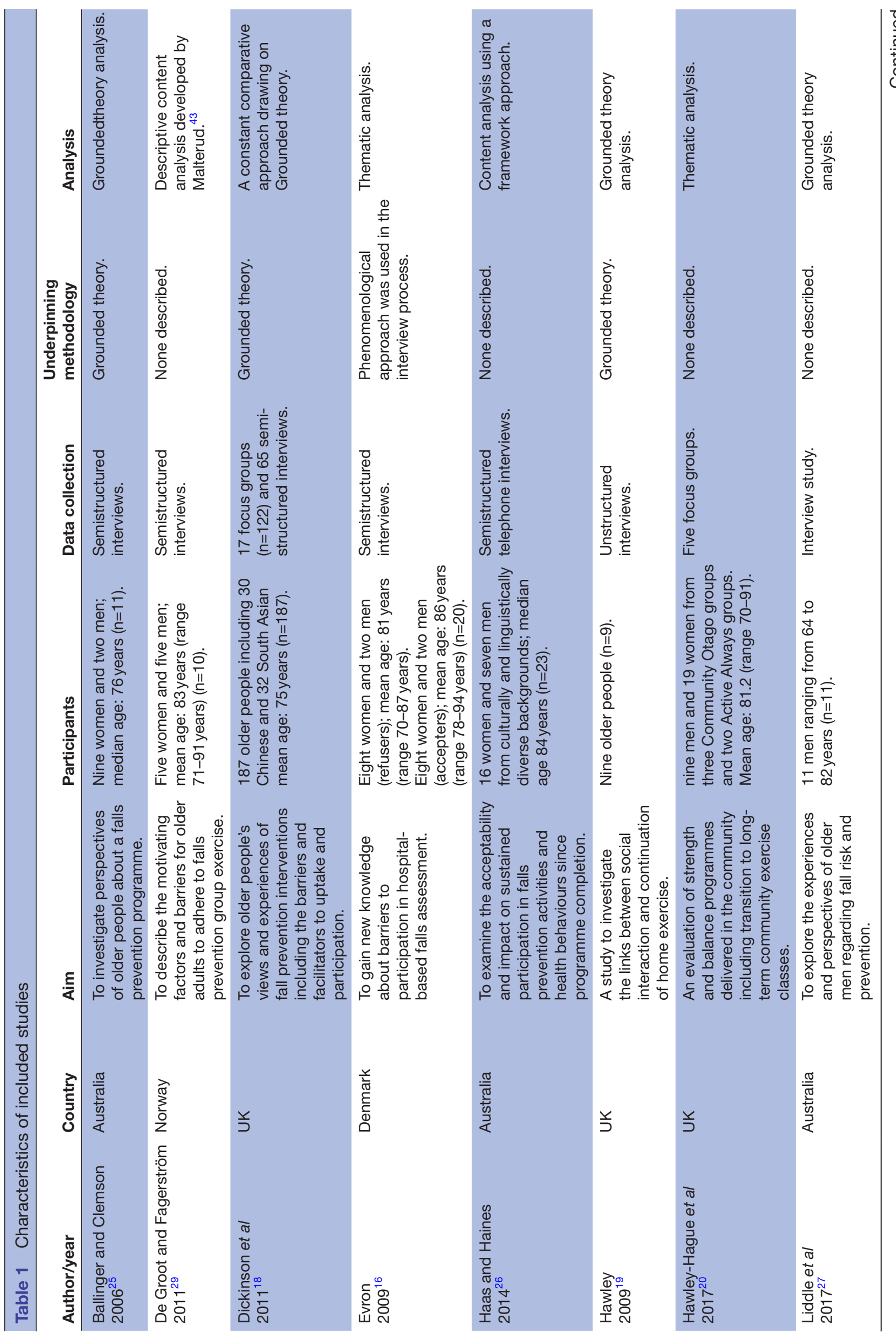




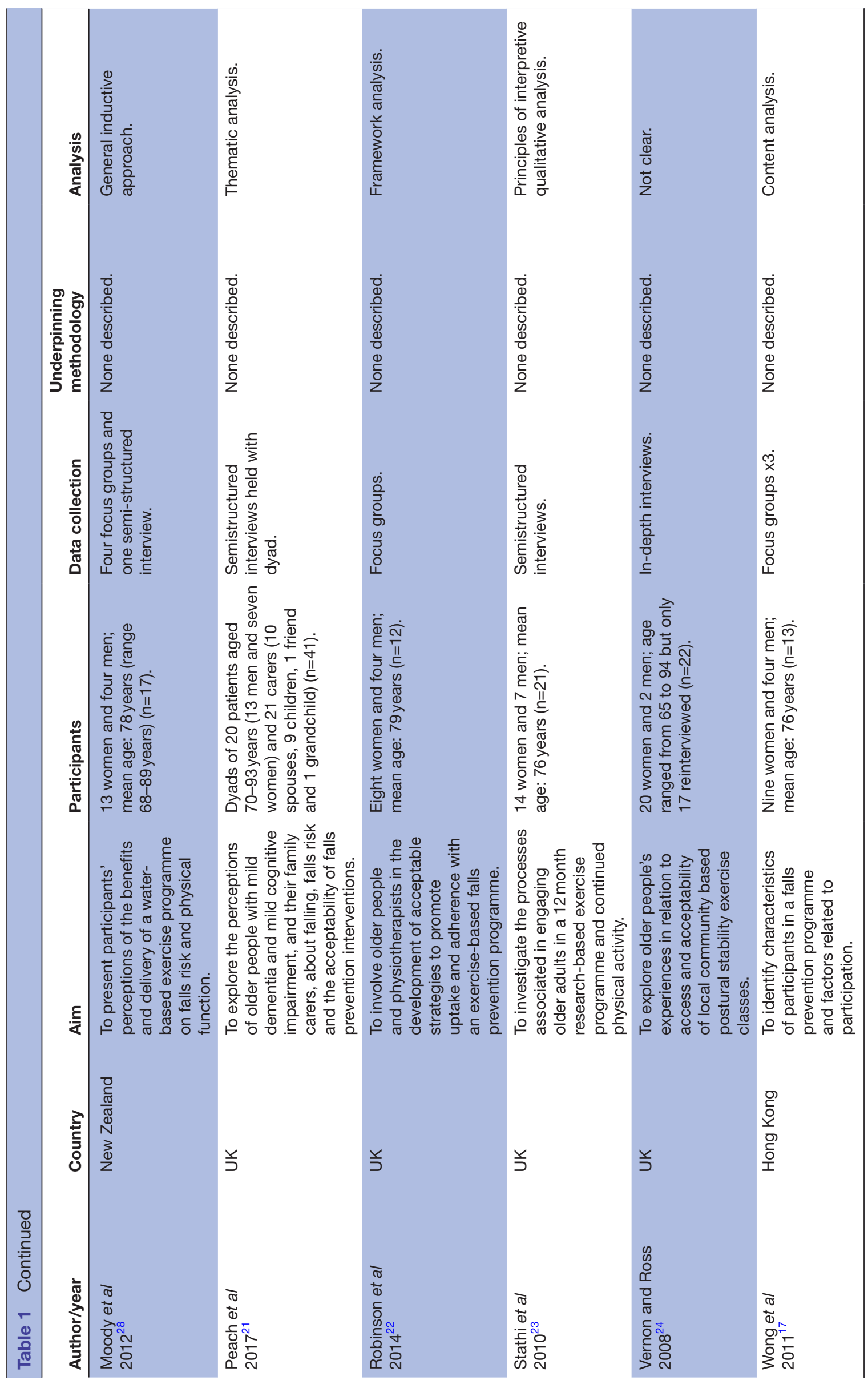




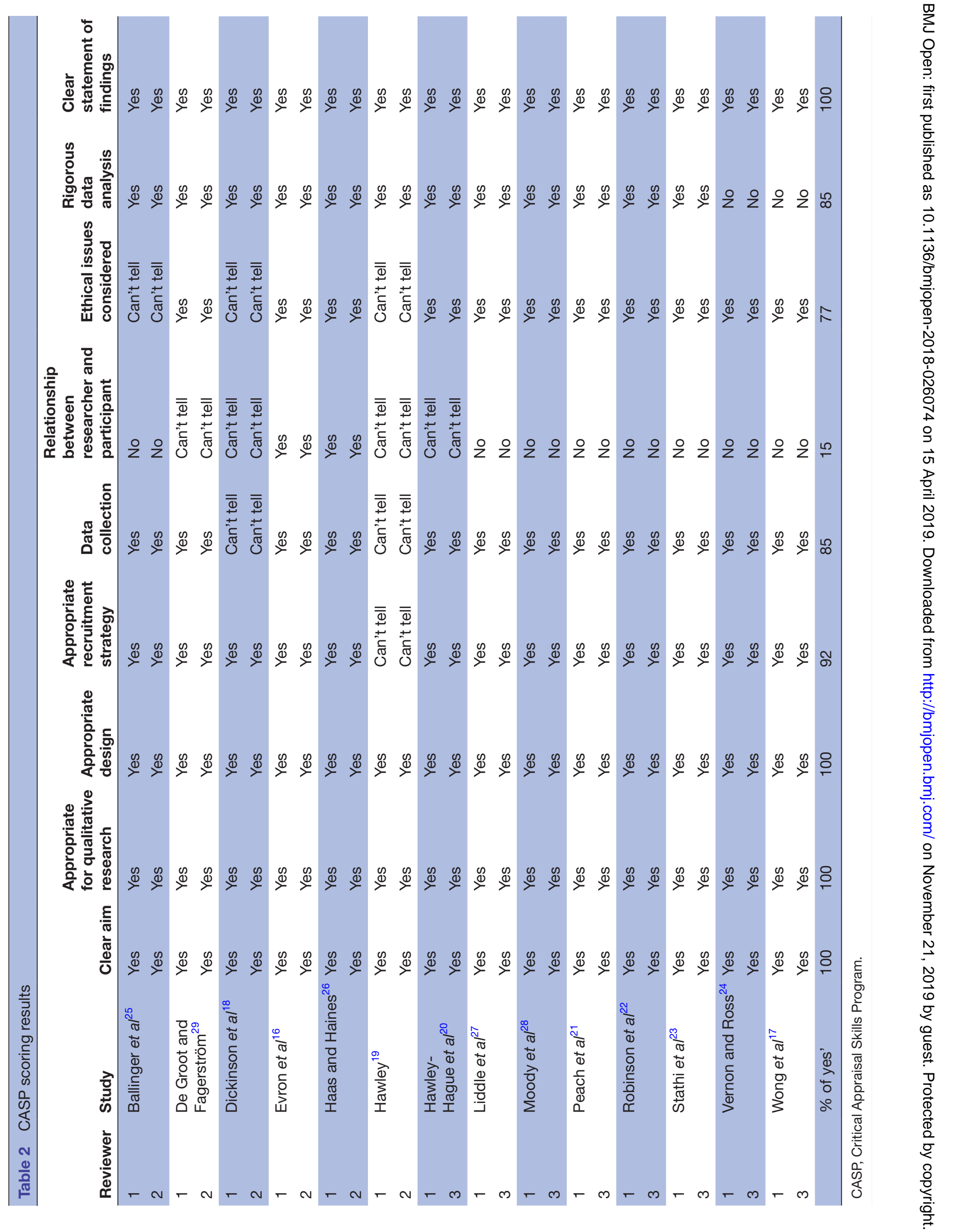


Table 3 GRADE-CERQual summary of qualitative findings table

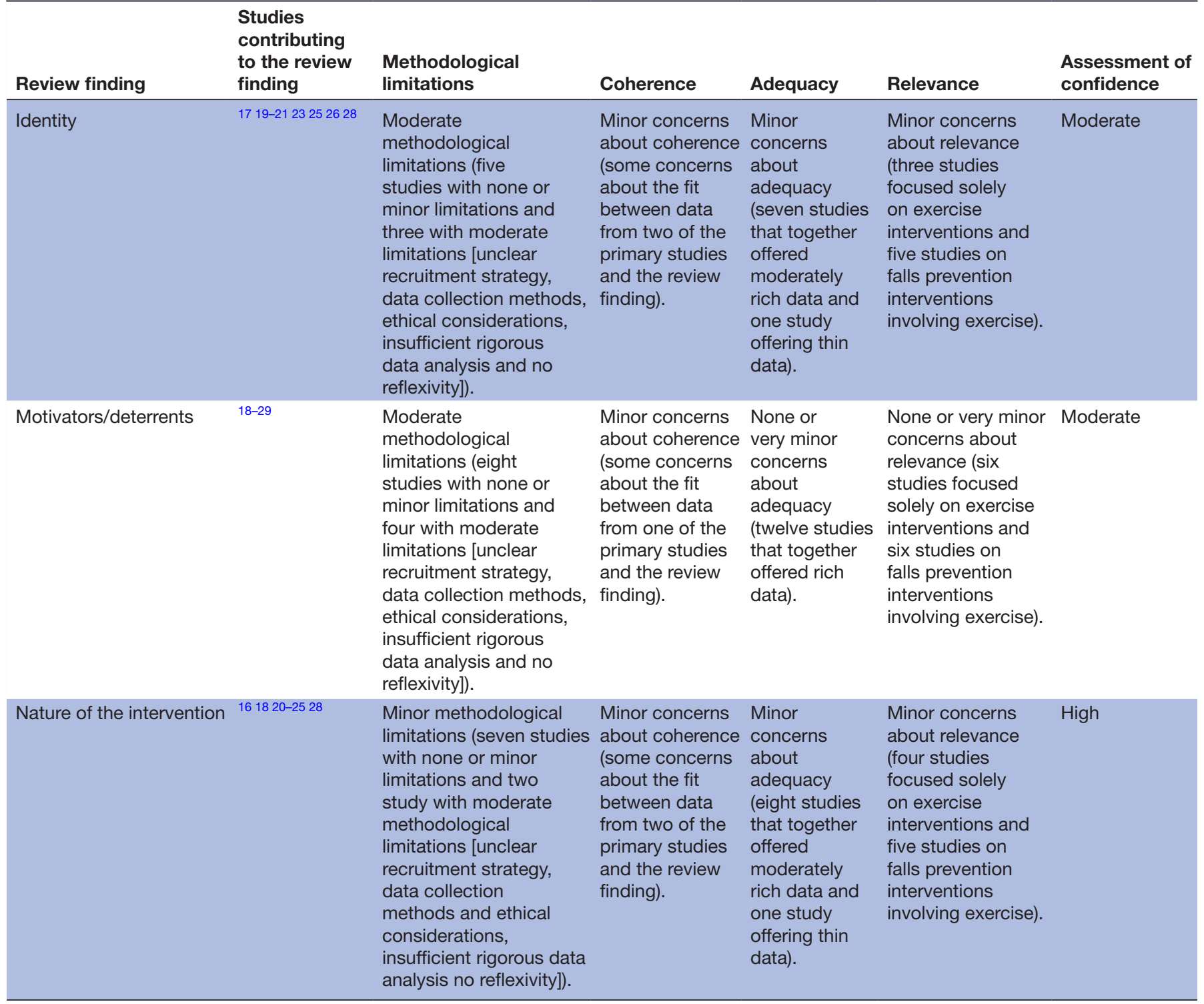

CERQual, Confidence in the Evidence from Reviews of Qualitative Research.

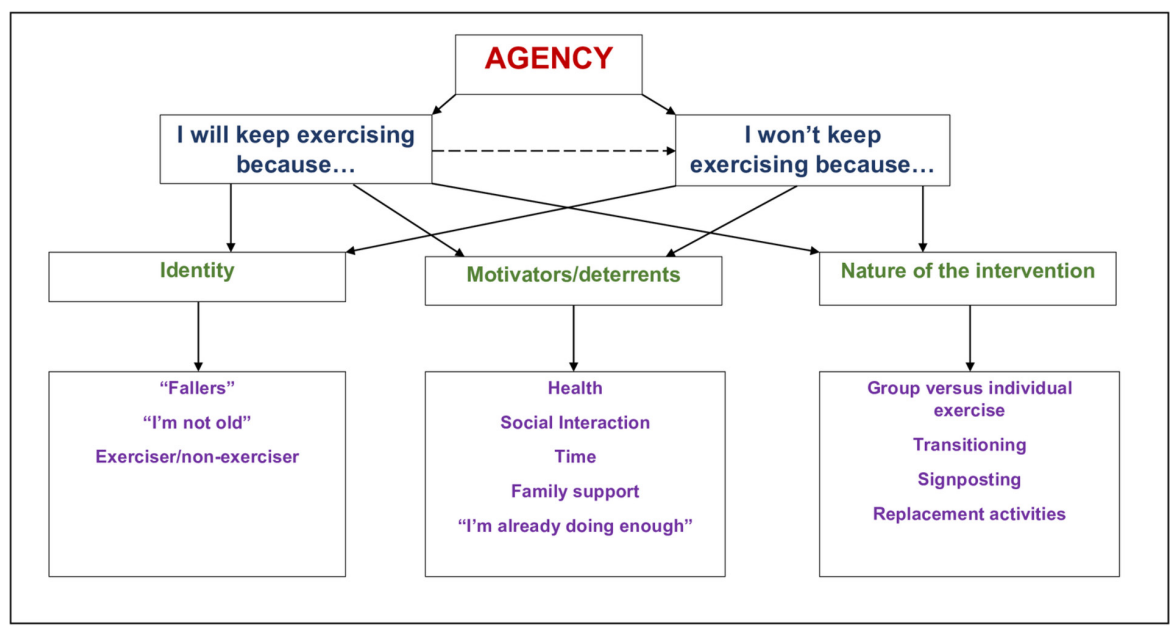

Figure 2 A diagram of descriptive and analytical themes. 
'They linked greater fall risk to getting old, but they did not see themselves as old. Being old was associated with the idea of not doing anything, an idea which the men rejected. Creating busy lives demonstrated they were not sitting around and therefore were not old ${ }^{27}$

\section{Exerciser/non-exerciser}

Older adults have a variety of attitudes and perceptions about what exercise means. The way they identified with exercise and their attitudes towards exercise impacted on how and why they either continued or discontinued with their exercises. The reasons to continue as an exerciser were for the perceived benefits to independence and general health and function. They also described an increase in confidence and ability to exercise because of taking part in their intervention:

'Participants are driven to be active in an attempt to achieve further independence or maintain the independence they have already achieved ${ }^{19}$

Intrinsic motivators predominantly revolved around the notion held by participants that exercise in general was good for them. They anticipated that the exercise would be beneficial to them for 'keeping fit', and 'keeping young' ${ }^{26}$

The improved levels of confidence and competence empowered some participants to meet the challenge of moving from a highly supervised environment to a more standard exercise environment ${ }^{23}$

Non-exercisers were able to clearly define reasons for no longer participating in exercise and justified this by labelling themselves to explain their reasons:

Those participants who were no longer exercising reported that they were too lazy, not in the right mood, no longer needed to exercise, did not enjoy exercising on their own or preferred another activity such as walking ${ }^{26}$

However, others gave a variety of reasons why they had found it difficult to maintain an exercise regime, including 'being the sort of person who hates exercise', ill health, lack of interest, being too busy and laziness $^{25}$

\section{Theme 2: motivators/deterrents}

This theme describes some of the issues that motivated participants to continue exercising after the end of the programme and in contrast, the deterrents which influenced some to stop exercising.

\section{Health}

Health benefits, maintaining health and preventing deterioration of health were all described as motivators to continued exercise. Preventing falls was seldom mentioned as a health benefit or motivator:

A number of functional health benefits were reported by the older people as a result of participating in an exercise-based falls prevention programme and these motivated them to continue with the prescribed exercise-regime $^{22}$

When patients who became active were asked about their motivation to change their behaviour, the leading response was their own belief that exercise was important to their health ${ }^{19}$

Conversely, health issues were also cited as a barrier to ongoing exercise:

One barrier to ongoing participation was that of illness $^{28}$

The physical barriers were health-related factors leading to dizziness, feeling shaky, pain, drowsiness, and reduced strength and endurance. The psychological barriers described were depression... ${ }^{29}$

\section{Social interaction}

Linked closely to an individual's preferences for group exercise, the social benefits of participating in group exercise frequently acted as a facilitator to continued participation, even though the transition to new groups could prove challenging. The social element related to being in the company of others, support from other group members and the instructor and the pleasure and enjoyment that this brought with it:

For some participants, social interaction was the main goal.... A social setting is an important factor to stimulate exercise on a regular basis ${ }^{29}$

The reasons given for continuing to exercise included... appreciation of the sociability involved in exercising $^{24}$

From community Otago to long-term maintenance in an Active Always class, it was...often a lot of the same group members which made participants feel more reassured and comfortable ${ }^{20}$

Participants exercising independently missed social interaction:

Continuing to do exercises at home independently was often discussed as being less enjoyable ${ }^{26}$

\section{Time}

Time was a recurring factor affecting long-term commitment to exercise. As a barrier, participants described a lack of time or being too busy to continue to exercise:

Perhaps a more surprising reason for not attending an intervention was that older people, although retired, often had other commitments, so found it difficult to attend regular interventions. Time pressures, due to caring responsibilities were another constraint ${ }^{18}$

Men who had stopped doing the exercises cited competing activities that took priority ${ }^{27}$

Conversely, for those who did continue to exercise, participants found ways to overcome issues with time by 
integrating their exercises into everyday life and fitting them into their routine; this acted as an important facilitator:

Incorporating the exercise into their daily routine also proved a popular strategy among the older people $^{22}$

Others who maintained their exercise regime adopted such strategies as viewing the exercises as a necessary regular chore and repeatedly reminding themselves of the obligation to carry them out, so that they felt guilty if the routine was missed ${ }^{25}$

...changes in their everyday schedule to accommodate the new and more active lifestyle ${ }^{23}$

\section{Family support}

As well as encouragement from their exercise instructors, participants described support and encouragement from others, usually family members. This was a facilitator to ongoing exercise:

This support was either family or spouse support, someone within the home encouraging them to keep going or close family support encouraging them to be active ${ }^{19}$

For the participants who had finished the course... one person repeated some of the exercises with another friend from the group. Another person... had a carer who helped her do the exercises at home... ${ }^{24}$

However, the 'support' of family members sometimes became a barrier to ongoing exercise as the responsibility became too burdensome for carers. Another issue was relatives' concerns for safety which led to activity restriction and was a major source of frustration for participants:

Several relatives described trying to prompt patients to exercise, but without success. Indeed, the need to remind patients, or monitor their exercise engagement, could constitute an unwelcome obligation ${ }^{21}$

Although most participants felt that they were supported by professionals or family to be active, some participants did find that at times they were restricted. This tended to be when concern for safety turned into restrictive behaviour. This restriction of activity led to feelings of frustration and a sense of loss... ${ }^{19}$

\section{'I'm already doing enough'}

Participants were committed to ongoing exercise until they felt their health status had improved to the level they perceived to be successful or they had achieved the goals that were meaningful to them, after which their commitment waned:

Previously practiced exercises were recognised to have been beneficial, but were discontinued once the need had passed, or only practised occasionally, as the habit had 'just drifted off somewhere' or exercise sheets were 'filed away'. Participants reported that they were currently 'doing enough' exercise through 'normal' activity... 21

...the reason for this lack of adherence to home exercise was that a level of independence was reached at which the benefits of home exercise no longer traded off against the risks (eg, fear of falling, injury, pain) ${ }^{19}$ ...related to this was the perception that the participant was already fit, strong and healthy and that their existing physical activities were sufficient to maintain this $^{26}$

\section{Theme 3: nature of the intervention}

This theme describes the practical and real-world experiences of older people participating in falls prevention exercise programmes.

\section{Group-based programmes versus individual exercise}

All the included studies involved older people who had participated in structured group exercise hence it was not possible to make comparisons with interventions directed at individuals exercising independently. For some, being part of a group was a facilitator to continue to exercise.

...exercising together with others gave them pleasure and had motivated them to continue exercising in the municipality ${ }^{29}$

For the participants who had finished the course of exercise classes, one person repeated some of the exercises they had been taught along with another friend from the group ${ }^{24}$

However, the group environment made some older adults feel uncomfortable and uneasy. For some, the experience of group exercise was a barrier to ongoing participation after completion of the programme:

Despite such observations, however, the older people indicated that they preferred to exercise at home ${ }^{22}$

Barriers were... unpleasant experience during previous exercise group sessions ${ }^{29}$

\section{Transitioning}

Despite their wish to continue to exercise, at the end of group interventions, some participants described the difficulty in transitioning from one exercise format to another. This included transitioning from their existing established group to a new exercise group or moving to exercising independently. For those keen to continue with group exercise, the main barriers to ongoing participation were identifying an appropriate local group to attend, difficulty obtaining information about new exercise opportunities and potential financial implications:

Difficulties were also caused when a fixed programme of exercise came to an end. Many participants who attended regularly exercise-based falls interventions found them to be beneficial and would have liked the classes to have continued for longer or to have had 
the opportunity to join a follow-on class. Often there were no suitable classes to which people could progress which affected motivation ${ }^{18}$

They were put off by not knowing where to access appropriate exercise in the community, or because they felt awkward and out of place in public places: e.g. feeling 'like an old lady' in the gym ${ }^{21}$

They did not want to use money on exercise programmes... ${ }^{16}$

\section{Signposting}

This transitional period over to a new exercise group highlights the significant role of the instructor or healthcare professional (HCP) leading the programme, not only during the intervention but as it comes to an end. The provision of appropriate, up-to-date and relevant information about other groups or new exercise opportunities was considered a facilitator:

The provision of informational support in the form of 'exit routes' to other exercise opportunities facilitated them to continue exercise. They praised the exercise class leaders' support during the transitional phase, and the available information about exercise choices in their local community ${ }^{23}$

...that ongoing input from a healthcare professional in an educational or advisory capacity would enhance feelings of self-efficacy and promote ongoing adherence $^{22}$

...the health professional suggested that the participant could maintain their exercises by attending a Community Otago class. Motivation to attend could also be related to an existing relationship that had been established with the instructor... this relationship helped transfer through from rehabilitation into long-term maintenance ${ }^{20}$

\section{Replacement activities}

For those who were unable to find a new group or those wishing to exercise independently, they sometimes took it on themselves or worked with their instructors to find practical ways to continue exercising. This did mean, however, that not everyone continued with the specific falls prevention exercises they were prescribed, choosing to exercise in different ways:

Participants seemed more likely to walk as a regular form of ongoing exercise than performing exercises specific to falls prevention. This was perhaps because walking was seen as functional and necessary (for example, to get to/from the shops) $)^{26}$

Participants referred to engaging in a wide range of leisure activities and hobbies which often involved activity and social engagement [including bowls, visiting friends and family, trips out, holidays, sewing, gardening, golf, walking, doing crosswords and puzzles, going into town, bus trips, reading, dancing, church-going $]^{21}$

\section{Analytical themes}

One overarching analytic theme encapsulating these descriptive themes emerged from the studies of older adults' views: agency. Agency can be defined as an individual's ability to act independently and to make their own free choices and decisions. ${ }^{30}{ }^{31}$ This ability is influenced by the individual's belief structure which has formed through their own aims and experiences as well as the views and perceptions of society and the structure of the environment that the individual is in. ${ }^{30} 32$

Therefore, in this instance, the participants' agency influenced their decision to continue exercising. Using their agency, these older people established their own identity and ascertained their own motivators or deterrents to continued exercise based on the nature of the exercise intervention.

\section{DISCUSSION}

Uptake of and adherence to falls prevention exercise programmes is often poor and research has been undertaken to establish the reasons for this. ${ }^{933}{ }^{334}$ However, there is lack of evidence about the barriers and facilitators to ongoing exercise when falls prevention programmes come to an end. It is important to consider these factors to promote behaviour change over the longer term.

This review systematically searched and synthesised the literature to identify perceptions of older people who have completed falls prevention exercise programmes. The analytical and descriptive themes identified provide a deeper understanding of what motivated or discouraged them from continuing exercising. All the included studies involved older adults who had participated in group exercise, thus, the review findings apply to this population.

The analytical theme emerging from this review is agency; that is an individual's ability to choose whether to continue to exercise based on their own beliefs, experiences and those of society and the environment they are in. ${ }^{30-32}$

The choice to continue to exercise after participation in a falls prevention exercise programme appears to be a dichotomy; participants either do exercise, or they don't. Although, as a caveat to this and highlighted in the 'I'm already doing enough' theme, after time, some people who do continue initially, stop once they achieve what they perceive to be successful personal goals. ${ }^{1921}$

Opting to continue to exercise seems to be an active process and older adults construct their own story as to why it is important for them to continue..$^{92326} \mathrm{In}$ contrast, discontinuing tends to be a more passive process; although older people still create their own story, and do not necessarily choose to be passive but exercise is just not a priority for them. ${ }^{25}{ }^{26}$ Frequently, despite recognising the potential benefits of exercising, those who stop exercising had a clear rationale for doing so including 'health issues', 'too lazy', 'hate exercise' or 'too busy'. To make exercise more of a priority for these 'non-exercisers' and 
to continue to motivate 'exercisers', HCPs need to understand all of their stories.

Older people have a clear sense of identity. Our findings are consistent with previous studies, highlighting that older adults feel that negative images and stereotypes often related to old age and falling did not fit with their identity. ${ }^{35-37}$ Instead, they perceive themselves as independent and capable people who can care for themselves, are not and do not wish to be reliant on or become a burden to others and act accordingly to maintain their independence. ${ }^{2527}$ Older people use their agency in deciding how to maintain their independence and these decisions may or may not include exercising. ${ }^{38}$

Thus, our review highlights a potential mismatch between priorities of the older person compared with those of HCPs delivering falls prevention interventions which typically involve strength and balance exercises. Rather than specifically preventing falls (goal of HCPs), most older adults reported being motivated to exercise to maintain or prevent deterioration of physical and mental health and remain functionally independent. ${ }^{1729}$ However, although willing to continue to exercise, older adults frequently opt for other forms of exercise after group programmes. ${ }^{21}{ }^{26} \mathrm{~A}$ potential reason for this is the lack of availability of appropriate community based groups to move on to. ${ }^{18-21} 2328$ Alternatively, as they do not identify themselves as 'fallers', they stop their strength and balance exercises and replace these with walking, as this is perceived to be more important for maintaining functional independence. ${ }^{172}$ This supports earlier work by McInnes and colleagues. ${ }^{33}$ HCPs need to promote ongoing strength and balance exercises as another way of meeting desired goals ensuring that older people understand the reasons for and potential benefits of these specific exercises during the supervised exercise phase. Commonly identified motivators to continued exercise such as maintaining independence ${ }^{19} 2629$ and social interaction/engagement ${ }^{19} 202429$ should be used by HCPs to promote continued exercise; appealing to these motivators while finding strategies to overcome barriers, especially during the transition period at the end of structured programmes.

This review highlights that behaviour change is an important factor to consider in the promotion of lifelong exercise. However, very few falls prevention programmes incorporate motivational-volitional behaviour change techniques and there is some uncertainty about which strategies might motivate older adults to undertake or continue with falls prevention exercises. ${ }^{39} 40$

There are a few feasibility studies of falls prevention exercise programmes incorporating behaviour change strategies. Fleig and colleagues ${ }^{39}$ used habit formation and helped participants with goal setting and action planning to facilitate ongoing exercise. At 6 months, participants showed an increase in action control (awareness of standards, self-monitoring and self-regulatory effort), action planning and automaticity (the degree to which the behaviour becomes habitual). They also reported an appreciation for interdisciplinary nature of the programme, and information on motivational techniques and habit formation.

McMahon and colleagues ${ }^{41}$ combined motivational and physical components of a falls prevention programme. The motivational component including social network support, motivational support and empowering education resulted in significantly improved behaviour and health outcomes in the intervention group. These studies show the potential benefit of incorporating behaviour change techniques into falls prevention exercise programmes, but larger UK based clinical trials, with adequate follow-up, are needed to provide evidence of long-term effectiveness.

This review highlights the importance of HCPs getting to know the stories of individuals participating in falls prevention exercise programmes. Similar to studies exploring uptake and adherence to exercise,${ }^{42}$ this review revealed that choosing to continue or stop exercising was influenced by a combination of factors including identity, health, social interaction and type of exercise. These findings give meaning to the participants' agency, encapsulating both the barriers and facilitators to ongoing exercise. It is important to identify these motivators and deterrents during the intervention phase and behavioural change strategies appear to have potential, alongside educational elements in the programmes, to help participants incorporate exercise into everyday life.

\section{Strengths and limitations}

To the best of our knowledge, this is the first systematic review of qualitative data examining the barriers and facilitators to ongoing falls prevention exercise after completion of an exercise programme.

Although we considered searching for grey literature, due to challenges of time this was not undertaken in a systematic manner. There is no main database for the identification of grey literature, unlike MEDLINE, EMBASE and other biomedical, social and psychological databases. We are familiar with internet-based information sites, including the System for Grey Literature in Europe which organises grey literature by subject area. However, these sites are challenging as they tend to lack advanced search and export features. Some also require subscription fees. We therefore acknowledge that this is a limitation of the review.

In relation to the synthesis, it is possible that other reviewers may have generated different themes, but the authors repeatedly read and reread the original studies to ensure themes were grounded in the data. To ensure trustworthiness in the data and findings of the review, CASP was used to assess transparency and relevance of the included studies and GRADE-CERQual tools were used to assess the credibility of our findings. Despite some methodological limitations in the primary studies, we have moderate to high confidence in the findings.

The descriptive and analytic themes illustrate what enables older people to continue with their falls prevention 
exercises. However, information about ongoing participation in exercise is limited as even in the included studies it was not the sole focus of the primary research. Since the participants in the included studies had all been through group-based falls prevention interventions there is a lack of information regarding older people's perspectives and experiences of individual (home-based) falls prevention exercise interventions. These people may have different experiences and, therefore, this is another area that needs further investigation.

\section{CONCLUSION}

Older people have their own individual and meaningful rationale for continuing or stopping exercise after the end of their falls prevention programme. Exploring these barriers and facilitators to continued exercise appears to be important during the intervention phase. It is important that HCPs get to know the older person's rationale and offer the best evidence-based practice and support to individuals, ensuring a smooth transition from structured interventions to independent exercise with sustained behaviour change. Enabling older people to continue with lifelong exercise requires understanding the place of exercise in their life and helping them to integrate it into their daily life, while keeping it relevant to them and their story.

Contributors SF, JB and KS conceived the idea for this systematic review. SF designed and conducted the literature searches. SF, JB and KS conducted data extraction, analysis and interpretation. SF wrote the first draft of the manuscript and all authors contributed to subsequent revisions and have agreed on the final version of the manuscript.

Funding The research was supported by The Dunhill Medical Trust (Ref No: RTF66/0116).

Competing interests None declared.

Patient consent for publication Not required.

Provenance and peer review Not commissioned; externally peer reviewed.

Data sharing statement № additional unpublished data are available.

Open access This is an open access article distributed in accordance with the Creative Commons Attribution Non Commercial (CC BY-NC 4.0) license, which permits others to distribute, remix, adapt, build upon this work non-commercially, and license their derivative works on different terms, provided the original work is properly cited, appropriate credit is given, any changes made indicated, and the use is non-commercial. See: http://creativecommons.org/licenses/by-nc/4.0/.

\section{REFERENCES}

1. WHO. Falls fact sheet. 2018 http://www.who.int/mediacentre/ factsheets/fs344/en/

2. Gillespie LD, Robertson MC, Gillespie WJ, et al. Interventions for preventing falls in older people living in the community. Cochrane Database Syst Rev 2012;9:CD007146.

3. Sherrington C, Tiedemann A, Fairhall N, et al. Exercise to prevent falls in older adults: an updated meta-analysis and best practice recommendations. N S W Public Health Bull 2011;22(3-4):78-83.

4. Lamb SE, Fisher JD, Gates S, et al. A national survey of services for the prevention and management of falls in the UK. BMC Health Serv Res 2008;8:233.

5. Nyman SR, Victor CR. Older people's participation in and engagement with falls prevention interventions in community settings: an augment to the Cochrane systematic review. Age Ageing 2012;41:16-23.
6. Hughes KJ, Salmon N, Galvin R, et al. Interventions to improve adherence to exercise therapy for falls prevention in communitydwelling older adults: systematic review and meta-analysis. Age Ageing 2018;0:1-11.

7. Robins LM, Hill KD, Day L, et al. Older Adult Perceptions of Participation in Group- and Home-Based Falls Prevention Exercise. J Aging Phys Act 2016;24:350-62.

8. McPhate L, Simek EM, Haines TP. Program-related factors are associated with adherence to group exercise interventions for the prevention of falls: a systematic review. J Physiother 2013;59:81-92.

9. Calhoun R, Meischke H, Hammerback K, et al. Older adults' perceptions of clinical fall prevention programs: a qualitative study. $J$ Aging Res 2011;2011:1-7.

10. Seers K. Qualitative systematic reviews: their importance for our understanding of research relevant to pain. $\mathrm{Br} J$ Pain 2015;9:36-40.

11. Thomas J, Harden A. Methods for the thematic synthesis of qualitative research in systematic reviews. BMC Med Res Methodol 2008;8:45.

12. Tong A, Flemming $\mathrm{K}$, Mclnnes $\mathrm{E}$, et al. Enhancing transparency in reporting the synthesis of qualitative research: ENTREQ. BMC Med Res Methodol 2012;12:181.

13. CASP Qualitative Checklist. 2018 https://casp-uk.net/wp-content/ uploads/2018/03/CASP-Qualitative-Checklist-Download.pdf.

14. Lewin S, Booth A, Glenton C, et al. Applying GRADE-CERQual to qualitative evidence synthesis findings: introduction to the series. Implement Sci 2018;13(Suppl 1):2.

15. Lewin S, Bohren M, Rashidian A, et al. Applying GRADE-CERQual to qualitative evidence synthesis findings-paper 2: how to make an overall CERQual assessment of confidence and create a Summary of Qualitative Findings table. Implement Sci 2018;13(Suppl 1):10.

16. Evron L, Schultz-Larsen K, Fristrup T. Barriers to participation in a hospital-based falls assessment clinic programme: an interview study with older people. Scand J Public Health 2009;37:728-35.

17. Wong EL, Woo J, Cheung AW, et al. Determinants of participation in a fall assessment and prevention programme among elderly fallers in Hong Kong: prospective cohort study. J Adv Nurs 2011;67:763-73.

18. Dickinson A, Machen I, Horton $\mathrm{K}$, et al. Fall prevention in the community: what older people say they need. $\mathrm{Br} J$ Community Nurs 2011;16:174-80.

19. Hawley H. Older adults' perspectives on home exercise after falls rehabilitation: Understanding the importance of promoting healthy, active ageing. Health Educ J 2009;68:207-18.

20. Hawley-Hague H, Roden A, Abbott J. The evaluation of a strength and balance exercise program for falls prevention in community primary care. Physiother Theory Pract 2017;33:611-21.

21. Peach T, Pollock K, van der Wardt V, et al. Attitudes of older people with mild dementia and mild cognitive impairment and their relatives about falls risk and prevention: A qualitative study. PLoS One 2017;12:e0177530.

22. Robinson L, Newton JL, Jones D, et al. Self-management and adherence with exercise-based falls prevention programmes: a qualitative study to explore the views and experiences of older people and physiotherapists. Disabil Rehabil 2014;36:379-86.

23. Stathi A, McKenna J, Fox KR. Processes associated with participation and adherence to a 12-month exercise programme for adults aged 70 and older. J Health Psychol 2010;15:838-47.

24. Vernon S, Ross F. Participation in community exercise classes: barriers to access. Br J Community Nurs 2008;13:89-92.

25. Ballinger C, Clemson L. Older People's Views about Community Falls Prevention: An Australian Perspective. British Journal of Occupational Therapy 2006;69:263-70.

26. Haas R, Haines TP. Twelve month follow up of a falls prevention program in older adults from diverse populations in Australia: a qualitative study. Arch Gerontol Geriatr 2014;58:283-92.

27. Liddle JL, Lovarini M, Clemson LM, et al. Men's perspectives on fall risk and fall prevention following participation in a group-based programme conducted at Men's Sheds, Australia. Health Soc Care Community 2017;25:1118-26.

28. Moody J, Hale L, Waters D. Perceptions of a water-based exercise programme to improve physical function and falls risk in older adults with lower extremity osteoarthritis: Barriers, motivators and sustainability. New Zealand Journal of Physiotherapy 2012;40:64-70.

29. de Groot GC, Fagerström L. Older adults' motivating factors and barriers to exercise to prevent falls. Scand J Occup Ther 2011;18:153-60.

30. Barker C. Cultural Studies. Theory and Practice. Third Edition. London: edSAGE Publications Ltd, 2008. 
31. Hojman DA, Miranda Álvaro. Agency, human dignity, and subjective well-being. World Dev 2018;101:1-15.

32. Alkire S. Dimensions of human development. World Dev 2002;30:181-205.

33. Mclnnes E, Askie L. Evidence review on older people's views and experiences of falls prevention strategies. Worldviews Evid Based Nurs 2004;1:20-37.

34. Horne M, Skelton D, Todd C. Attitudes and beliefs about the uptake and adherence of exercise and physical activity in $60-70$ year olds in relation to fall prevention. British Psychological Conference Proceedings of the British Psychological Society, Coventry:Coventry University, 2005.

35. Yardley L, Bishop FL, Beyer N, et al. Older people's views of fallsprevention interventions in six European countries. Gerontologist 2006;46:650-60.

36. Gardiner S, Glogowska M, Stoddart C, et al. Older people's experiences of falling and perceived risk of falls in the community: A narrative synthesis of qualitative research. Int J Older People Nurs 2017;12:e12151.

37. Mclnnes E, Seers K, Tutton L. Older people's views in relation to risk of falling and need for intervention: a meta-ethnography. J Adv Nurs 2011;67:2525-36.
38. Yardley L, Donovan-Hall M, Francis K, et al. Older people's views of advice about falls prevention: a qualitative study. Health Educ Res 2006;21:508-17.

39. Fleig L, McAllister MM, Chen P, et al. Health behaviour change theory meets falls prevention: Feasibility of a habit-based balance and strength exercise intervention for older adults. Psychol Sport Exerc 2016;22:114-22.

40. French DP, Olander EK, Chisholm A, et al. Which behaviour change techniques are most effective at increasing older adults' self-efficacy and physical activity behaviour? A systematic review. Ann Behav Med 2014:48:225-34.

41. McMahon SK, Wyman JF, Belyea MJ, et al. Combining Motivational and Physical Intervention Components to Promote Fall-Reducing Physical Activity Among Community-Dwelling Older Adults: A Feasibility Study. Am J Health Promot 2016;30:638-44.

42. Hawley-Hague H, Horne M, Skelton DA, et al. Older Adults' Uptake and Adherence to Exercise Classes: Instructors' Perspectives. $J$ Aging Phys Act 2016;24:119-28.

43. Malterud K. Kvalitative metoder I medisinsk forskning - En Mnnforing [in Norwegian] [Qualitative Methods in Medical Research - An Introduction. Universitets for laget, 2003. 